\title{
Monostotic fibrous dysplasia of the $\mathrm{C} 4$ cervical spine
}

\author{
M. Sambasivan, P. Sanalkumar, S. Mahesh, K. Rajasekharan Nair, Abeed Basheer \\ Cosmopolitan Hospitals (P) Ltd, Pattom, Trivandrum, Kerala, India
}

A 35-year-old male patient presented with pain in the back of the neck and right arm since eight months. He had been getting vague discomfort along the right arm since one year. Since two months he noticed tingling sensation on the right side of the body and fingers on bending or turning the neck to the right. No definite history of trauma was noted. Patient was not a diabetic or hypertensive. Patient gave no history of pulmonary tuberculosis.

On examination the $(\mathrm{O} / \mathrm{E})$ - Patient was conscious, responsive, oriented, right-handed. Speech was normal. No cranial nerve deficits. No motor deficits. No evidence of wasting of muscles. No fasciculations.

Deep tendon jerks were symmetrical. Plantars were equivocal on both sides. Visceral reflexes were normal.

Sensory system examination revealed - Dysasthesia over C5-C6 and C6-C7 distribution on the right side.

Examination of the cervical spine did not present any abnormality. No abnormal mass was palpated over the paraspinal region or over the spinous process.

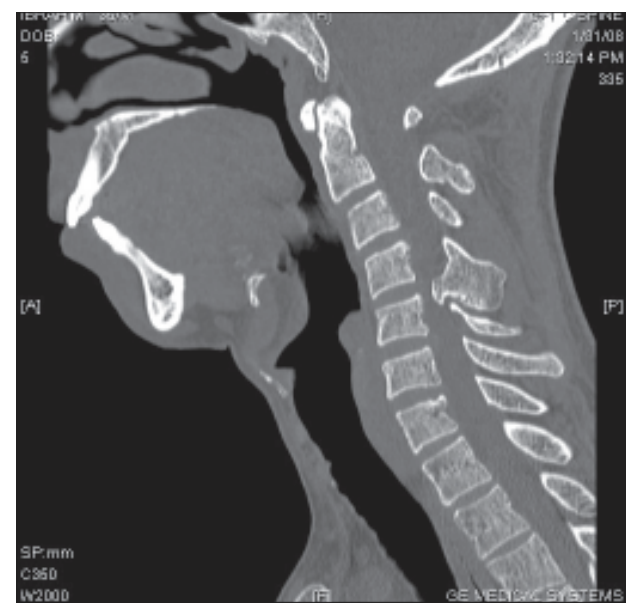

Figure 1: CT cervical spine showing fibrous dysplastic lamina complex of C4 (Bone window) vertebra encroaching into the cervical spinal canal
Right lateral flexion of the neck was restricted due to pain going down the right side of the body. Patient was investigated with Computed tomography and Magnetic resonance imaging (CT and MRI) scan of cervical spine.

CT cervical spine - An irregular bony growth was seen arising from the posterior neural arch of $\mathrm{C} 4$ vertebra projecting and compressing the spinal cord [Figures 1 and 2].

MRI scan of the cervical spine [Figures 3 and 4] - A bony outgrowth was seen arising from the spinous process and lamina of $\mathrm{C} 4$ vertebra projecting and compressing the spinal cord at that level. Cervical spinal cord appeared flattened. A provisional diagnosis of fibrous dysplasia/ osteoid osteoma was made.

At operation an abnormal bony mass was noticed at $\mathrm{C} 4$ spinous process and lamina extending more to the right side. Laminectomy of C4-C5 with excision of bony tumor was done. At the site of cord compression the dura appeared discolored. After decompression the dura was pulsating normally. The excised tumor was sent for histopathological examination and

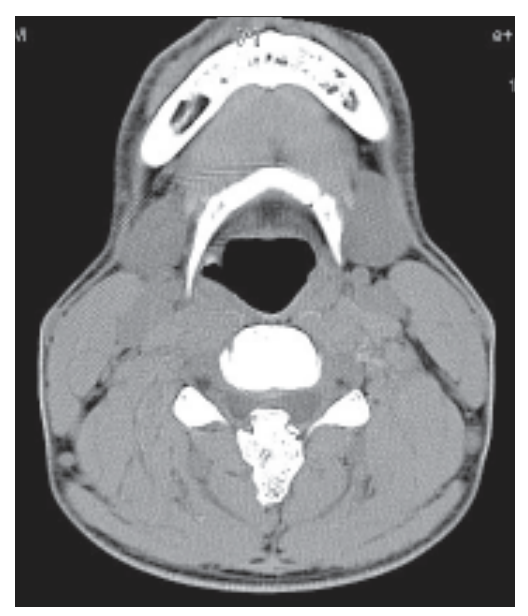

Figure 2: CT cervical spine ( transverse cut ) showing fibrous dysplasia of $\mathrm{C} 4$ lamina encroaching into the canal and compressing the cervical spinal cord 

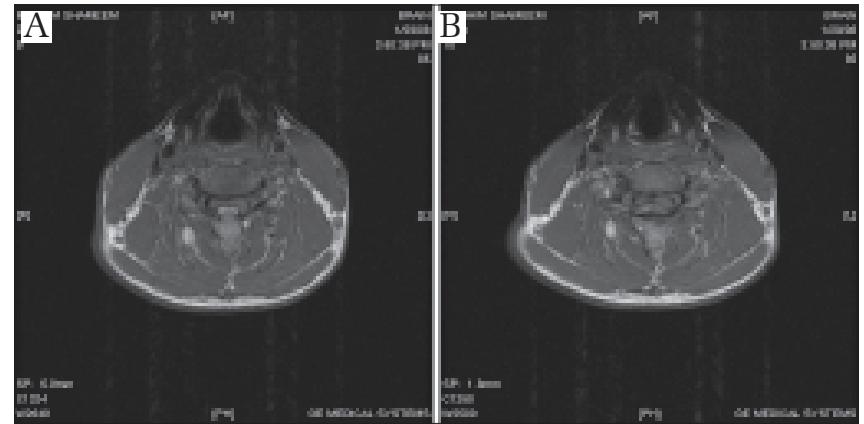

Figure 3A and B: MRI scan of cervical spine (transverse cut ) showing flattened cervical spinal cord due to compression by the fibrous dysplastic element of C4 cervical vertebra

the picture was consistent with fibrous dysplasia. Postoperatively the patient made a good recovery.

The term fibrous dysplasia was used first by Lichtenstein $^{[1]}$ in 1938 to designate a developmental anomaly of unknown etiology characterized by fibrous tissue replacement of medullary cavity of bones. Any bone in the body can be involved. The involvement can be either monostotic or polystotic. Monostotic fibrous dysplasia of the spine is a rare entity. Only 26 cases, of which 11 were located in the cervical spine, are to be found in the literature. A case of monostotic fibrous dysplasia of the cervical spine was published by Proschek et al.,${ }^{[2]}$ in the Archives of orthopedic and trauma surgery on 2007 . This article is the latest one to our knowledge. Our report describes the $12^{\text {th }}$ patient who had a monostotic fibrous dysplasia involving the neural arch of the fourth cervical vertebra. The patient made an uneventful recovery after excision of the lesion. At follow-up three weeks later the patient was absolutely symptom-free.
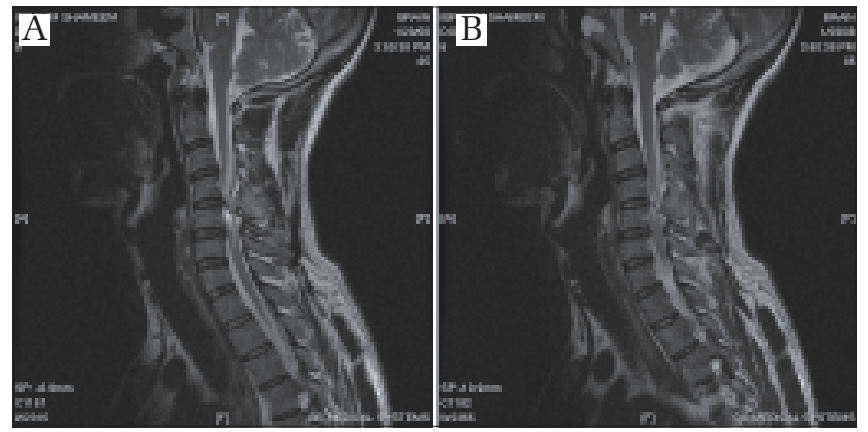

Figure 4A and B: MRI scan of cervical spine ( sagittal section ) showing the spinal cord compression by the posterior dysplastic lamina complex of C4 vertebra

\section{Acknowledgment}

We are greatly indebted to the directors and staff of the Cosmopolitan Hospital for helping and allowing us to publish this material. We are thankful to the Cosmopolitan Educational and Research Foundation for providing us with the material for researching this article. We are also thankful to Mr. M. Vijayachandran for his help in preparing the manuscript.

\section{References}

1. Lichtenstein L. Polystotic fibrous dysplasia. Arch Surg 1938;36:87798.

2. Proschek D, Orler R, Stauffer E, Heini P. Monostotic fibrous dysplasia of the spine - report of a case involving a cervical vertebra. Arch Orthop Trauma Surg 2007;127:75-9.

Accepted on $07-04-2008$

Source of Support: Nil, Conflict of Interest: None declared. 